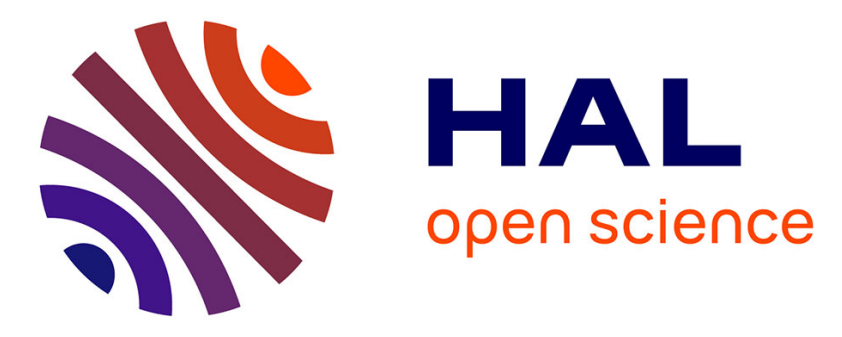

\title{
Probing Interconnectivity in Hierarchical Microporous/Mesoporous Materials using Adsorption and Nuclear Magnetic Resonance Diffusion
}

Anne Galarneau, Flavien Guenneau, Antoine Gedeon, Diaa Mereib, Jeremy

Rodriguez, François Fajula, Benoit Coasne

\section{To cite this version:}

Anne Galarneau, Flavien Guenneau, Antoine Gedeon, Diaa Mereib, Jeremy Rodriguez, et al.. Probing Interconnectivity in Hierarchical Microporous/Mesoporous Materials using Adsorption and Nuclear Magnetic Resonance Diffusion. Journal of Physical Chemistry C, 2016, 120 (3), pp.1562-1569. 10.1021/acs.jpcc.5b10129 . hal-01384395

\section{HAL Id: hal-01384395 \\ https://hal.science/hal-01384395}

Submitted on 15 Feb 2021

HAL is a multi-disciplinary open access archive for the deposit and dissemination of scientific research documents, whether they are published or not. The documents may come from teaching and research institutions in France or abroad, or from public or private research centers.
L'archive ouverte pluridisciplinaire HAL, est destinée au dépôt et à la diffusion de documents scientifiques de niveau recherche, publiés ou non, émanant des établissements d'enseignement et de recherche français ou étrangers, des laboratoires publics ou privés. 


\section{Probing Interconnectivity in Hierarchical Microporous/Mesoporous}

\section{Materials using Adsorption and Nuclear Magnetic Resonance Diffusion}

Anne Galarneau, ${ }^{\dagger} * *$ Flavien Guenneau, ${ }^{\dagger}$ Antoine Gedeon, ${ }^{\star}$ Diaa Mereib, ${ }^{\star}$ Jeremy Rodriguez, ${ }^{\dagger}$ François Fajula, ${ }^{\dagger}$ Benoit Coasne ${ }^{\dagger, \S, *}$

† Institut Charles Gerhardt Montpellier, UMR 5253 CNRS-Université de MontpellierENSCM, ENSCM, 8 rue de l’Ecole Normale, 34296 Montpellier Cedex 05, France

† Sorbonne Universités, UPMC Univ Paris 06, CNRS, Collège de France, UMR 7574, Laboratoire de Chimie de la Matière Condensée de Paris, 4 place Jussieu, Case 196, 75252 Paris Cedex 05, France

$\S$ MultiScale Materials Science for Energy and Environment (UMI 3466 CNRS/MIT) and Department of Civil and Environmental Engineering, Massachusetts Institute of Technology, Cambridge, MA 02139, United States.

* To whom correspondence should be sent.

A. Galarneau (anne.galarneau@enscm.fr), B. Coasne (coasne@mit.edu) 
Abstract. Adsorption and transport in hierarchical materials are investigated by means of adsorption and nuclear magnetic resonance experiments. Using micro/mesoporous zeolites with well-defined mesoporosity, we show that adsorption at a given pressure can be described as a simple linear combination of the adsorbed amounts taken at the same pressure for the pure microporous (zeolite FAU-Y) and mesoporous (Al-MCM-41) solids. Such a quantitative decomposition allows us to demonstrate the ability of diffusion measurements by Pulsed Field Gradient Nuclear Magnetic Resonance (PFG NMR) to probe interconnectivity in hierarchical solids. On the one hand, transport in the mechanical mixtures can be described as the superimposition of diffusion in pure microporous and mesoporous solids. On the other hand, PFG NMR for the hierarchical sample provides an effective diffusivity that is intermediate between those for the pure zeolite and mesoporous silica. Furthermore, this effective diffusivity is slower than the linear combination of the two diffusivities weighted by the number of molecules occupying each phase (used in the independent domain and fastexchange theories) clearly showing interconnectivities and transfer limitations between the microporous and mesoporous domains. We also discuss the ability of combining theories such as the fast exchange model and the effective medium theory to quantitatively predict diffusion in such microporous/mesoporous materials. 


\section{Introduction}

Owing to their unique properties such as their microporous crystalline network and large surface area, zeolites are at the heart of key applications in catalysis, adsorption, ionexchange, etc. ${ }^{1}$ However, the small pores and aperture sizes in zeolites $(<1 \mathrm{~nm})$ lead to mass transfer limitations and reduced access to the active surface while preventing desorption of bulky products (the latter induce pore blocking which eventually leads to zeolite deactivation). To circumvent these issues, important efforts are devoted to the design of hierarchical materials combining porosity scales such as micro and mesoporosities., ${ }^{2,3}$ Starting from a zeolite, mesoporosity is added using post-synthesis treatments based on steaming, acid leaching, and desilication. ${ }^{4}$ Such treatments generate a broad mesopore distribution that does not allow optimal control of active site access with a precise contact time. More ordered mesoporosities can be generated by adding surfactants during zeolite synthesis (comparable to the synthesis of ordered mesoporous silica but taking zeolite as silica source). However, depending on the zeolite, the additional mesopores are either connected to the microporosity or restricted to the external layer of the zeolite crystal (in some cases, an additional MCM-41 powder appears as a separate product with no connection to the zeolite). ${ }^{2}$ Great performance enhancements in catalysis have been obtained thanks to such mesoporous zeolites. ${ }^{5,6}$ While further work is needed to clarify the effect of loading (including low loadings which can be relevant for specific applications), we expect that diffusivity increases such as that observed in the present work is general and applies to most realistic process conditions.

While the benefits of combining porosities have been demonstrated (Refs. 5, 7, and 8 for instance), the design of such hierarchical solids still relies on trial and error strategies. Only true hierarchical zeolites with interconnected micro/mesopores enhance mass transfer. Ideally, 
the mesoporosity should also percolate through the zeolite crystal to ensure direct access from the external phase to the core of the zeolite particles. There is therefore a strong motivation to develop simple methods to probe interconnectivity in hierarchical materials. For instance, Garcia-Martinez et al. provided evidence of the hierarchical nature of micro/mesoporous Faujasite (hereafter mFAU) using electron tomography. ${ }^{9}$ By means of argon adsorption at 65 $\mathrm{K}$ these authors also showed that only a very small part of the mesoporosity (representing $0.08 \mathrm{~mL} / \mathrm{g}$ ) is not accessible from the external surface. Pulsed Field Gradient Nuclear Magnetic Resonance (PFG NMR) is a robust and efficient method to assess interconnectivity in micro/mesoporous materials by determining the self-diffusivity of a confined adsorbate. ${ }^{10,11,12}$ PFG NMR showed that additional mesoporosity $(20 \mathrm{~nm})$ in a MCM-41 (3.7 $\mathrm{nm}$ ) increases hexane diffusivity by a factor of 3.7 if such large mesopores are connected to the external surface (a very limited effect is observed if they are not directly accessible ${ }^{13}$ ). This is supported by the fact that diffusion in dealuminated zeolite, known to possess isolated mesopores, is close to that in regular zeolite. ${ }^{10}$ As another example of the complex effect of additional porosity in micro/mesoporous zeolites, no drastic changes were observed for ethane diffusivity in hierarchical LTA, ${ }^{14}$ due to the very fast diffusivity of this molecule, while the diffusivity of propane increased drastically with additional mesoporosity. The diffusivity increased with the increase of mesoporous volume and could be enhanced by a factor of 100 in comparison with pristine LTA crystals. ${ }^{15,16}$ Similarly, cyclohexane diffusivity in hierarchical mesoporous $\mathrm{NaX}^{15}$ was found to increase by a factor 5 .

Despite its robustness, the interpretation of PFG NMR data relies on the validity of phenomenological models developed to estimate the effective diffusivity in heterogeneous media through combination rules, which are sometimes presented as analogies of electric transport in resistance circuits. The fast-exchange model $^{17}$ assumes that hierarchical transport 
does not involve energy barriers between porosity scales so that ergodicity holds (i.e. the mean residence time $t_{k}$ in a porosity type $k$ is proportional to the average fraction $x_{k}$ of molecules in this porosity). The fast exchange model usually overestimates the measured effective diffusivity $D_{\text {eff }}$ as it favors the fast diffusivity contribution (large porosity) by assuming that $D_{\text {eff }}$ corresponds to the linear combination of the diffusivities $D_{k}$ weighed by their population $x_{k}$. In contrast, by assuming that the diffusivity combines as in electrical resistors $R_{k}$ in series $\left(R_{k}=1 / D_{k}\right), \quad D_{\text {eff }}$ usually underestimates the experimentally measured diffusivity as it favors the slow diffusivity contribution (small porosity scale). Recently, Bonilla et al. ${ }^{18}$ used the Effective Medium Theory ${ }^{19}$ (EMT) which predicts that $D_{\text {eff }}$ obeys Bruggeman's combination rule ${ }^{20}$ (initially derived for dielectric constants in composite media). While EMT predicts $D_{\text {eff }}$ that is closer to the experimental value because it lies between the lower and upper boundaries predicted using the two models above, questions about the analogy between electrical phenomena in composite media and fluid transport in multiscale porous media remain open; non trivial effects such as surface barriers, which cannot be captured using the approaches above, add up to the intrinsic complexity of hierarchical transport. For instance, using an empirical relation for surface diffusion, it was proposed that only $\sim 1$ out of 2000 pore openings in a microporous Metal Organic Framework (Zn(tbip)) are accessible from the external surface. ${ }^{21,22}$ However, this will depend on the postmodification of the microporous sample; for instance, dealuminated zeolites can have some of their pores coated by alumina debris if no further washing is performed.

Here, we report an in-depth analysis of the hierarchical interconnectivity in micro/mesoporous Faujasite (mFAU) using hexane adsorption and diffusion experiments. PFG NMR ability to probe such interconnectivity is assessed by comparing the data for mFAU with those for regular Faujasite zeolite and for ordered mesoporous silica Al-MCM-41. A mechanical 
mixture of FAU zeolite and Al-MCM-41 with the same ratio of microporous and mesoporous volumes as in mFAU, but with no hierarchy since no connections between the micro- and mesopores are present, is also considered. Thanks to the use of model materials with welldefined porosities, we show that adsorption can be described using a superimposition principle; i.e. the adsorbed amount at a given pressure can be obtained by a linear combination of the adsorption isotherms for the parent solids (zeolite and Al-MCM-41) where the weighing parameters are the volume fractions occupied by each phase. This result shows that adsorption does not allow distinguishing a true hierarchical material from a material made of independent micro and mesoporosities. In contrast, PFG NMR is shown to allow probing interconnectivity unambiguously in such hierarchical micro/mesoporous zeolites.

\section{Experimental section}

2.1. Materials synthesis. $\mathrm{Al}-\mathrm{MCM}-41(\mathrm{Si} / \mathrm{Al}=15)$ materials were synthesized by adding $\mathrm{NaAlO}_{2}$ (Carlo Erba) in an alkaline solution containing octadecyltrimethylammonium bromide surfactant $\left(\mathrm{C}_{18} \mathrm{TAB}\right)$ (Aldrich). The mixture was stirred at $323 \mathrm{~K}$ until complete dissolution before adding silica Aerosil 200 (Degussa) and stirring for $1 \mathrm{~h}$. The composition of the mixture in molar ratio is: $1 \mathrm{SiO}_{2} / 0.07 \mathrm{NaAlO}_{2} / 0.1 \mathrm{C}_{18} \mathrm{TAB} / 0.125 \mathrm{NaOH} / 50 \mathrm{H}_{2} \mathrm{O}$. The slurry was then placed in a stainless autoclave at $423 \mathrm{~K}$ for $20 \mathrm{~h}$. The resulting material was then filtered and washed until neutral $\mathrm{pH}$, dried at $353 \mathrm{~K}$, and calcined at $823 \mathrm{~K}$ for $8 \mathrm{~h}$ under air-flow.

Hierarchical micro/mesoporous FAU (mFAU) was prepared from dealuminated H-FAU-Y $(\mathrm{Si} / \mathrm{Al}=15)$ named CBV720, which was purchased from Zeolyst. This zeolite was chosen to keep the same $\mathrm{Si} / \mathrm{Al}$ ratio as in $\mathrm{Al}-\mathrm{MCM}-41$ in order to ensure the closest surface chemistry for both materials. This zeolite was slightly dealuminated by the supplier to reach $\mathrm{Si} / \mathrm{Al}=15$, 
which creates at the same time some additional large mesopores (diameters $>30 \mathrm{~nm}$ ) visible in the high-pressure region of the nitrogen adsorption isotherm (not shown). However, this will not affect our study as the mesoporosity generated using the pseudomorphism transformation with octyltrimethylammonium $\left(\mathrm{C}_{18} \mathrm{TAB}\right)$ in basic medium leads to much smaller mesopores around $4 \mathrm{~nm}$. The pseudomorphic synthesis is a technique that mimicks natural transformations in the mineral world, where another crystalline structure is formed in the habit of the native one by slow dissolution and recrystallization processes. By adapting the dissolution rate of a crystal to the crystallization rate of another crystalline phase, the morphology of the pristine crystals is maintained while the crystalline structure is changed. This has been successfully applied to silica particles in order to form MCM-41 particles of defined sizes. ${ }^{23}$ The same technique was used with FAU-Y particles ${ }^{9}$ in the presence of quaternary ammonium surfactant and $\mathrm{NaOH}$ in order to form mesoporous FAU with MCM41 like mesoporosity.

The micropore volume of the parent H-FAU-Y material, $0.36 \mathrm{~mL} / \mathrm{g}$ (determined by nitrogen adsorption at $77 \mathrm{~K}$ ), corresponds to that expected for pure crystalline Faujasite. In order to prepare mFAU, an alkaline solution was prepared with $\mathrm{C}_{18} \mathrm{TAB}$ in sodium hydroxide solution at $323 \mathrm{~K}$ under stirring. After complete dissolution of $\mathrm{C}_{18} \mathrm{TAB}$ in $\mathrm{NaOH}$ solution, the FAU zeolite powder was added and stirred for $1 \mathrm{~h}$. The composition of the mixture in molar ratio corresponds to: $1 \mathrm{Si} / 0.07 \mathrm{Al} / 0.1 \mathrm{C}_{18} \mathrm{TAB} / 0.125 \mathrm{NaOH} / 50 \mathrm{H}_{2} \mathrm{O}$. The slurry was then placed in a stainless autoclave at $423 \mathrm{~K}$ for $24 \mathrm{~h}$. The resulting material was then filtered and washed until neutral $\mathrm{pH}$, dried at $353 \mathrm{~K}$, and calcined at $823 \mathrm{~K}$ for $8 \mathrm{~h}$ under air-flow.

2.2. Materials characterization. $X$-Ray Diffraction (XRD) patterns of the materials were measured using a Bruker D8 Advance diffractometer with a Bragg-Brentano geometry and 
equipped with a Bruker Lynx Eye detector. XRD patterns were recorded in the range 4-50 (20) to identify zeolite peaks and in the range $1-5^{\circ}(2 \theta)$ to detect the low angle XRD peaks corresponding to the mesopores. The angular step size was $0.0197^{\circ}$ and the counting time $0.2 \mathrm{~s}$ per step. The textural properties of the materials were determined from the $\mathrm{N}_{2}$ adsorption/desorption isotherms at $77 \mathrm{~K}$ measured on a Micromeritics Tristar 3000 apparatus. The samples were previously outgassed in vacuum at $523 \mathrm{~K}$ for $12 \mathrm{~h}$. The mesopore diameter was estimated using the Broekhoff-De Boer method as it is considered one of the most accurate methods for pore size determination in the case of mesoporous silica. ${ }^{24}$ The mesoporous volume was taken at $P / P_{0} \sim 0.5$ (after capillary condensation). For hierarchical micro/mesoporous materials, the microporous and mesoporous volumes were calculated by $\mathrm{t}-$ plot analysis using a home-made reference isotherm ${ }^{25}$ and using the proposed abacus to correct t-plot analysis for microporous volume determination. ${ }^{25}$ Particle morphology was studied using a Hitachi S-4500 I scanning electron microscope (SEM). Particle size distributions were determined by granulometric analysis (Mastersizer 2000 from Malvern Instruments) using an optimized procedure for MCM-41 samples obtained by pseudomorphic transformation. ${ }^{23} 200 \mathrm{mg}$ of sample was added to $10 \mathrm{~mL}$ acetone, ultrasonicated for $30 \mathrm{~min}$, and dried before being dispersed into $700 \mathrm{~mL}$ of water for analysis. Before hexane adsorption, samples were dehydrated at $523 \mathrm{~K}$ under vacuum. Hexane adsorption isotherms were measured at $298 \mathrm{~K}$ using a homemade adsorption equipment from 0 to 100 Torr.

2.3. Pulsed Field Gradient Nuclear Magnetic Resonance (PFG NMR). For diffusion studies we chose to adsorb an amount of hexane corresponding to $60 \%$ of the total adsorption capacity. The desired adsorbate quantity was obtained by introducing the required pressure in a calibrated volume of the adsorption apparatus. This amount was subsequently trapped by freezing in the NMR sample tube containing the solid. Before PFG-NMR measurements, 
samples were kept at room temperature for at least one night to guarantee total adsorption equilibrium. The PFG experiments were run on a $300 \mathrm{MHz}$ Bruker DSX spectrometer equipped with a Diff50 probe delivering a maximum gradient of $30 \mathrm{~T} \mathrm{~m}^{-1}$. Following our previous work on diffusion in large crystals of $\mathrm{NaX}$ zeolites, ${ }^{26}$ the bipolar 13 -interval pulse sequence $^{27}$ was chosen to avoid any effect arising from the presence of internal field gradients. In this case, the echo attenuation, $\Psi=I / I_{0}$, takes the following form (adapted from $\left.\operatorname{ref}^{27}\right)$ :

$$
\Psi=\exp \left[-\gamma^{2} \delta^{2} g^{2} D_{e f f}\left(\Delta+\frac{\tau}{4}-\frac{\delta}{6}\right)\right]
$$

where $D_{\text {eff }}$ is the effective diffusion coefficient, $\Delta$ the observation time i.e. the time interval between the gradient pulses, $\gamma$ the gyromagnetic ratio, $\delta$ the duration of the gradient pulses, and $g$ the gradient strength. $\tau$ is the time interval between the last radiofrequency pulse and the spin echo (echo time). The pulse duration and the observation time were set to $1 \mathrm{~ms}$ and $10 \mathrm{~ms}$ respectively, and the echo time was $5.6 \mathrm{~ms}$. The intensity of the applied gradients was set between 0 and $2.1 \mathrm{~T} \mathrm{~m}^{-1}$. The duration of the $\pi / 2$ radiofrequency pulse was $10 \mu \mathrm{s}$. All the experiments were performed at $298 \mathrm{~K}$. Typically, $D_{\text {eff }}$ is obtained from the slope of the echo attenuation curves $\ln \Psi=f\left(g^{2}\right)$ which correspond to the linear transformation of Eq. (1).

\section{Results}

3.1. Adsorption. Hierarchical zeolite mFAU with mesopores diameter of $4.4 \mathrm{~nm}$ (characterized by nitrogen adsorption and XRD (Fig. S2 and S3)) has been synthesized with a higher amount of $\mathrm{NaOH}^{25}$ in comparison to the mFAU synthesis of Garcia-Martinez et al. ${ }^{9}$ in order to increase the mesoporous volume fraction and minimize the effect of not fully accessible mesopores on diffusion measurements. Figure 1 shows the hexane adsorption isotherms at $298 \mathrm{~K}$ for FAU, Al-MCM-41 and mFAU. Both Al-MCM-41 and mFAU exhibit 
a type IV adsorption isotherm, characteristic of mesoporous materials, with a sharp increase at $P \sim 30$ Torr revealing capillary condensation in the mesoporosity. The fact that capillary condensation occurs for both materials at the same pressure indicates that the mesoporosity in these two samples is similar. The total pore volume of $4.58 \mathrm{mmol} / \mathrm{g}(\sim 0.60 \mathrm{~mL} / \mathrm{g})$, as determined from the density $\rho=0.6548 \mathrm{~g} / \mathrm{mL}$ of bulk hexane at $298 \mathrm{~K}$, is in agreement with that estimated from nitrogen adsorption at $77 \mathrm{~K}: 0.68$ and $0.64 \mathrm{~mL} / \mathrm{g}$ for Al-MCM-41 $(4.1 \mathrm{~nm}$ pore diameter) and mFAU (4.4 $\mathrm{nm}$ pore diameter), respectively (Fig. S2). In contrast to mFAU and Al-MCM-41, FAU exhibits a type I adsorption isotherm, which is characteristic of microporous samples. The pore volume $1.54 \mathrm{mmol} / \mathrm{g}(\sim 0.20 \mathrm{~mL} / \mathrm{g})$ determined from such a hexane adsorption isotherm is lower than the micropore volume determined by nitrogen adsorption at $77 \mathrm{~K}(0.36 \mathrm{~mL} / \mathrm{g})$. This is due to the fact that part of the microporosity of Faujasite, the sodalite cages volume (aperture $0.22 \mathrm{~nm}$ ), is not accessible to hexane due to its size $(0.420 \mathrm{~nm})$; the supercage volume features $0.283 \mathrm{~mL} / \mathrm{g}$. It has to be noted that the pore volume of FAU determined in this study $(0.20 \mathrm{~mL} / \mathrm{g})$ using hexane adsorption is in good agreement with previous results from the literature $(0.16-0.27 \mathrm{~mL} / \mathrm{g}$ depending on the FAU post-treatments ${ }^{28}$ ). Consequently, only the supercages of FAU are filled with hexane.

With the aim to test the validity of PFG NMR to probe diffusion in hierarchical micro/mesoporous materials, we first determined with accuracy the amounts of hexane confined in each porosity scale. To do so, we used the linear combination method that was recently proposed ${ }^{29,30}$ in which the hexane adsorption isotherm $n(P)$ (in mmol per $\mathrm{g}$ of sample) for mFAU is expressed as the sum of the adsorption isotherms for the pure microporous solid, $f_{\text {micro }}(P)$, and for the pure mesoporous solid, $f_{\text {meso }}(P)$ :

$$
n(P)=\left\lfloor V_{\text {micro }}^{\text {ph }} f_{\text {micro }}(P)+V_{\text {meso }}^{\text {ph }} f_{\text {meso }}(P)\right\rfloor \rho
$$


where $\rho=7.61 \mathrm{mmol} / \mathrm{mL}$ is the bulk number density of hexane at $298 \mathrm{~K}$ and the weighing parameters $V_{\text {micro }}^{p h}$ and $V_{\text {meso }}^{p h}$ are the volumes occupied by the microporous and mesoporous phases respectively, expressed in $\mathrm{mL}$ per $\mathrm{g}$ of sample. $f_{\text {micro }}$ and $f_{\text {meso }}$, which are the reference adsorption isotherms for the microporous and mesoporous solids, are expressed as the fraction of the phase volume (i.e. not only the porous volume but also the matrix volume) being filled at a pressure $P$. Figure 1 shows that Eq. (2) provides an excellent fit for the hexane adsorption isotherm of mFAU. The volumes of the microporous and mesoporous phases are found to be $V_{\text {micro }}^{p h}=0.15$ and $V_{\text {meso }}^{p h}=0.50 \mathrm{~mL} / \mathrm{g}$, respectively. This result is in good agreement with nitrogen adsorption data analyzed by corrected t-plot analysis $(0.12$ and $0.52 \mathrm{~mL} / \mathrm{g}){ }^{25}$ Therefore, for $100 \%$ hexane loading (100 Torr, $4.94 \mathrm{mmol} / \mathrm{g}$ ) in mFAU, there are 1.14 and $3.80 \mathrm{mmol} / \mathrm{g}$ of hexane in the microporosity and in the mesoporosity, respectively.

3.1. Diffusion. PFG NMR has proven to be a valuable tool to gain insight into the porosity of various materials, through the characterization of the diffusing properties of adsorbed probe molecules. However, the measurement of genuine intracrystalline diffusion coefficients requires that the molecules remain in the intraparticle space during the whole NMR pulse sequence. With this regard, PFG NMR measurements benefit from the large size of the particles of FAU, mFAU and Al-MCM-41 centered at 6, 34 and 69 microns, respectively (Fig. S4). These particles are intergrowths of crystallites of a size $1 \mu \mathrm{m}$, generating intercrystallite pores of ca. $0.3 \mu \mathrm{m}$ (Fig. S5). As previously shown, ${ }^{13}$ only particles with mean size above several $\mu \mathrm{m}$ could be studied by PFG NMR in our conditions as n-hexane diffusion is otherwise too fast considering the experimental observation times attainable (more than $6 \mathrm{~ms}$ in our case). This leads to a fast signal attenuation essentially characteristic of longrange diffusion in the interparticle space. In order to minimize the effect of hexane adsorbed 
at the external surface of the micron-sized particles, PFG NMR measurements were performed at a loading of $60 \%$ for each material (FAU, Al-MCM-41, mFAU and a mechanical mixture of FAU/Al-MCM-41). As explained above, thanks to the decomposition of the adsorption isotherm for mFAU as a linear combination of the adsorption isotherms for FAU and Al-MCM-41, we are able to estimate for a given pressure or loading the corresponding adsorbed amount in the microporosity and mesoporosity of mFAU. For a loading of $60 \%$, we found that there are $0.9 \mathrm{mmol} / \mathrm{g}$ adsorbed in the microporosity and 1.85 $\mathrm{mmol} / \mathrm{g}$ in the mesoporosity (the corresponding mole fractions in the micro and mesoporosities are $x_{\mu}=0.33$ and $x_{m}=0.67$, respectively).

Figure 2 shows the spin echo attenuation curves for hexane adsorbed at $298 \mathrm{~K}$ in the different materials for $\Delta=10 \mathrm{~ms}$. Clearly, Eq. (1) doesn not describe adequately the experimental data and one has to consider a superimposition of exponential functions:

$$
\Psi(\Delta)=\sum_{i=1}^{n} p_{i} \exp \left[-(\gamma \delta g)^{2} D_{i}\left(\Delta+\frac{\tau}{4}-\frac{\delta}{6}\right)\right]
$$

where $p_{i}$ denotes the relative number of molecules having a diffusion coefficient $D_{i}$. From Figure 2 one can suspect two distinct components, i.e. $n=2$ in Eq. (3), for most of the samples under study. Since the different diffusion coefficients $D_{i}$ appear to be sufficiently separated, two independent linear relationships were used to describe the diffusion data. The initial steep decay is attributed to molecules leaving the porous particles and diffusing rapidly in the interparticle space. The second part of the attenuation represents the diffusion of n-hexane strictly confined in the mesoporous and/or microporous network. It was previously shown for FAU-X particles formed of intergrowths of FAU-X crystallites that this diffusivity does not represent the genuine intracrystalline diffusion but rather the intraparticle diffusion (intracrystalline diffusion within different crystallites of the same particle hindered by diffusion barriers between crystallites). ${ }^{26,31,32}$ By rationalizing the effect of these barriers in 
the theoretical framework of restricted diffusion, i.e. by measuring the effective diffusion coefficient as a function of the observation time, the authors showed that water diffusion was 3 times slower in the intraparticle regime than in the purely intracrystalline regime, due to transport resistance at the interface between individual crystallites and their external surface. ${ }^{32}$ Thanks to the similar particle morphology of FAU, mFAU and Al-MCM-41 (Fig. S5), the intraparticle diffusivities measured in this study are expected to be influenced to the same extent by diffusion barriers. No dependence of the diffusion coefficients on the observation time could be detected within the experimental accuracy for $\Delta$ values between 7 and $50 \mathrm{~ms}$. Moreover, pseudomorphic transformation allows us to transform FAU into mFAU and in the same time maintains the morphology of the crystallite clusters. The two intraparticle diffusion coefficients can therefore be directly compared as they are similarly affected by aggregation and diffusion barriers between crystallites. The corresponding effective diffusion coefficient $D_{\text {eff }}$ was estimated by fitting $\ln \Psi$ as a function of $g^{2}$ against Eq. (3) in the high field gradient range (Figure 2). Figure 3 shows the diffusion coefficients for the different systems; $D_{\text {eff }}=$ $2.24 \pm 0.50 \times 10^{-10} \mathrm{~m}^{2} / \mathrm{s}$ for FAU, $1.23 \pm 0.10 \times 10^{-8} \mathrm{~m}^{2} / \mathrm{s}$ for Al-MCM-41, $1.38 \pm 0.1 \times 10^{-9} \mathrm{~m}^{2} / \mathrm{s}$ for mFAU. As expected, the diffusivity in the mesoporous material is much faster than in the microporous material. Such a diffusion enhancement, by roughly two orders of magnitude, is well documented in the literature for materials exhibiting a single porosity scale..$^{11,33,34,35,36}$

The PFG NMR experiment for the mechanical mixture (FAU + Al-MCM-41) with the same amount of micro and mesoporosity as in mFAU can be described with the help of Eq. (3) by setting the number of components to $n=3$. In addition to the fast decaying component, this gives two distinct effective diffusion coefficients, $D_{\text {eff }}^{\text {fast }}=1.38 \pm 0.1 \times 10^{-8} \mathrm{~m}^{2} / \mathrm{s}$, which is close to that measured for Al-MCM-41, and $D_{\text {eff }}^{\text {slow }}=1.87 \pm 0.50 \times 10^{-10} \mathrm{~m}^{2} / \mathrm{s}$ which is close to that measured for FAU. This result is due to the fact that, in such a mechanical mixture, molecular 
exchange between the different porosities is very limited on the typical time scale probed by PFG NMR, and since it will proceed through the interparticular space, the NMR signal from the exchanging molecules will only contribute to the fast decaying part of the attenuation curve. As a result, one does not observe an "average" effective diffusivity as in mFAU but rather the superimposition of the contribution of molecules diffusing solely in the microporous or in the mesoporous material.

The additional mesoporosity in mFAU increases by a factor 6.2 the diffusion of hexane, from a very slow diffusion regime for FAU $\left(2.2 \times 10^{-10} \mathrm{~m}^{2} / \mathrm{s}\right)$ to a faster diffusion regime for $\mathrm{mFAU}$ $\left(1.4 \times 10^{-9} \mathrm{~m}^{2} / \mathrm{s}\right)$. This result is fully consistent with previous data obtained for hierarchical NaX zeolite. ${ }^{14,15}$ The diffusion coefficient for hexane in mFAU is found to be intermediate between those for the pure microporous and mesoporous materials. While the diffusion coefficient for such micro/mesoporous materials will be discussed in detail in the next section, the results above already show that PFG NMR allows probing unambiguously interconnectivity in hierarchical solids. Indeed, in contrast to adsorption which does not allow distinguishing true hierarchical materials from mixtures of microporous and mesoporous powders, the effective diffusivity as probed by PFG NMR exhibits a value that deviates from those observed for pure microporous and mesoporous samples and for their mixture.

\section{Discussion}

As discussed in the introduction, several phenomenological models are available in the literature to interpret the self-diffusivity in heterogeneous porous media. These models, which are analogies to electrical transport in resistor circuits, provide combining rules to estimate the 
effective diffusivity $D_{\text {eff }}$ based on the diffusivities $D_{k}$ for the different porous domains $k$. Despite interesting successes, the validity of such approaches could not be fully established in the case of micro/mesoporous materials due to the lack of accurate estimates of the adsorbed amounts in each porosity scale. Moreover, these models do not constitute a unifying and comprehensive theory of hierarchical transport as they correspond to different physical pictures without clear indication about their range of validity. For instance, as discussed by Kärger and coworkers, ${ }^{17}$ the fast exchange model is expected to be physically relevant when no surface diffusion barriers are involved between the different porosity domains. In contrast, while surface barriers can be taken into account empirically, their insertion in a multiscale model of transport in hierarchical materials remains to be achieved. In what follows, we consider these different models and discuss their validity in the light of our experimental measurements reported in the previous section. Thanks to the use of a hierarchical material with well-defined micro and mesoporosity, we are able to confront the different models with our experimental diffusion data since the adsorbed amount in each porosity scale is accurately known from the linear combination method discussed in Section 3.1.

Figure 4 compares the self-diffusivity obtained from PFG NMR and the values predicted using the different models. The fast-exchange model has been used by many authors to interpret PFG NMR data on micro/mesoporous materials. In this model, one considers a medium made of $\mathrm{M}$ domain types (corresponding to microporous or mesoporous domains for instance). The self-diffusivity is related to the average mean square displacement $\left\langle\Delta r^{2}\right\rangle=\left\langle(\boldsymbol{r}(t)-\boldsymbol{r}(0))^{2}\right\rangle$ in the Fickian regime $\left(\left\langle\Delta r^{2}\right\rangle \propto t\right)$ :

$$
D_{\text {eff }}=\lim _{t \rightarrow \infty} \frac{1}{6 t}\left\langle(\boldsymbol{r}(t)-\boldsymbol{r}(0))^{2}\right\rangle=\lim _{t \rightarrow \infty} \frac{1}{6 t}\left\langle\left(\sum_{k=1}^{M} \sum_{i} \Delta r_{i}^{k}\right)^{2}\right\rangle
$$


In the right-hand side, we wrote that $\Delta r$ is the sum of each molecule displacement $\Delta r_{i}^{k}$ along its $i^{\text {th }}$ trajectory segment in a domain of type $k$. If path $i$ in a domain $k$ is assumed to be uncorrelated with any of the paths in a different domain type $k \neq k$, the sum over the M domains can be extracted from the average < > in Eq. (4):

$$
D_{e f f}=\lim _{t \rightarrow \infty} \frac{1}{6 t} \sum_{k=1}^{M}\left\langle\left(\sum_{i} \Delta r_{i}^{k}\right)^{2}\right\rangle
$$

By definition, for each domain $k,\left\langle\left(\sum_{i} \Delta r_{i}^{k}\right)^{2}\right\rangle=6 t_{k} D_{k}$ ( $t_{\mathrm{k}}$ is the typical time spent in a porous domain $k$ ) so that Eq. (5) can be rewritten:

$$
D_{e f f}=\sum_{k=1}^{M} \tau_{k} D_{k}=\sum_{k=1}^{M} x_{k} D_{k}
$$

where $\tau_{k}$ is the average fraction of time spent by a molecule in a domain $k$. The right-hand side of Eq. (6) is obtained by assuming that the average time $\tau_{k}$ is equal to the average fraction of molecules in domains $k$ at a given time (ergodicity principle). Using the self-diffusion coefficient $D_{k}$ and mole fractions $x_{k}$ for each domain type $k$ (see above), the fast-exchange (FE) model predicts $D_{\text {eff }}^{\mathrm{FE}}=8.3 \times 10^{-9} \mathrm{~m}^{2} / \mathrm{s}$ which overestimates by a factor $\sim 6$ the effective diffusivity of hexane in mFAU observed using PFG NMR (Figure 4). While the fast-exchange model is often used to interpret diffusion data in heterogeneous media, our results show that it does not describe accurately transport in hierarchical porous media such as mesoporous zeolites. The combination of the two diffusivities weighed by the number of molecules in each phase is also equivalent to the calculation of the average diffusivity in totally independent domains. Therefore, the lower diffusivity obtained by PFG NMR for mFAU indicates non negligible interconnectivity with transfer limitation between the two porosities in this sample. 
As discussed in Refs. 17 and ${ }^{37}$, there are well-known situations where the breakdown of the fast-exchange model is expected. The fast-echange model assumes perfect stochasticity; this implies that, once a molecule leaves a porosity domain $k$ for a porosity domain $k$, the probability that it returns to this domain type is equal to the probability to remain in the domain type $k^{\prime}$. As discussed in Ref. 17, it is clear that this assumption fails for layered materials made of domains of different types placed in series. In the latter case, the selfdiffusivity is expected to be given by the following combination rule:

$$
\frac{1}{D_{\text {eff }}}=\sum_{k=1}^{M} \frac{x_{k}}{D_{k}}
$$

which corresponds to the combination rule for resistors, having a resistance $R_{k}=1 / \mathrm{D}_{k}$, placed in series. Using Eq. (7), we obtain the "serial resistors" self-diffusivity $D_{\text {eff }}^{\mathrm{SR}}=6.5 \times 10^{-10} \mathrm{~m}^{2} / \mathrm{s}$ which drastically underestimates the self-diffusivity of hexane in mFAU observed using PFG NMR (Figure 4). As expected, the effective diffusivities predicted from the fast-exchange and serial resistors models provide upper and lower boundaries for the experimental diffusivity (due to the fact that the linear combination for the fast exchange model favors the fast diffusion contribution while that for the serial resistors model favors the slow diffusion contribution). Interestingly, this result suggests that a reasonable range estimate of the diffusion in hierarchical porous media can be obtained from these two values.

The results above show that transport in hierarchical porous media does not obey simple linear combination rules. In fact, while analogies to electrical transport are probably relevant, it is unclear what formalism should be adopted (such as series or parallel resistors for instance). The lack of unifying framework to describe the effective diffusivity in hierarchical porous media is consistent with the fact that the abundant literature on diffusion in microporous/mesoporous media refers to one model or the other without clear justification 
(most papers refer to the fast-exchange model and invoke diffusion barrier effects). In an attempt to accurately predict transport in these materials, Bhatia, Kärger and coworkers ${ }^{18}$ have proposed to use the Effective Medium Theory (EMT). Compared to the other models considered above, this theory also relies on an analogy with transport in electrical circuits but it does not assume an assembly of domains in series or in parallel but rather a connected network of domains. Within the framework of EMT, the effective self-diffusivity $D_{\text {eff }}$ is obtained from the following formula, known as Bruggeman's combination rule, which applies to the conductivities $k(\sim \rho D)$ :

$$
\sum_{k} \phi_{k} \frac{k_{k}-k_{e f f}}{k_{k}+\left(\frac{z}{2}-1\right) k_{e f f}}=0
$$

where $\phi_{k}$ is the volume occupied by the domains of type $k$ and $z$ is the network connectivity $(z$ $=6$ for a $3 \mathrm{D}$ network). $k_{k}$ corresponds to the conductivity of domain $k$ while $k_{\text {eff }}$ is the effective conductivity. The self-diffusivity $D_{k}$ within a domain $k$ is defined from Fick's law which assumes that the flux $J$ induced by a concentration gradient is directly proportional to this concentration gradient, $J_{k}=-D_{k} \nabla \mathrm{c}$. In the latter equation, $\nabla \mathrm{c}$ is the local concentration gradient within the domain which corresponds to a bulk concentration gradient $\nabla \mathrm{c}_{k}{ }^{\mathrm{b}}$ such that $\nabla \mathrm{c}=f_{k} \nabla \mathrm{c}_{k}^{\mathrm{b}}$ where $f_{k}=\partial \mathrm{c} / \partial \mathrm{c}_{k}^{\mathrm{b}}$ is the equilibrium constant. The single phase conductivities corresponds to $k_{\mathrm{k}}=f_{k} D_{k}$ while the effective conductivity is $k_{\text {eff }}=f_{\text {eff }} D_{\text {eff }}$ with the effective equilibrium constant $f_{\text {eff }}$ simply given by the combining rule $f_{\text {eff }}=\sum_{k} \phi_{k} f_{k}$. Using these definitions and taking $\mathrm{z}=6$, Eq. (8) can be recast to obtain the combination rule for the effective self-diffusivity:

$$
\sum_{k} \phi_{k} \frac{f_{k} D_{k}-f_{\text {eff }} D_{\text {eff }}}{f_{k} D_{k}+2 f_{\text {eff }} D_{e f f}}=0
$$


Solving Eq. (9) leads to $f_{\text {eff }} D_{\text {eff }}=-a / 4 \pm\left[a^{2}-8 b\right]^{1 / 2} / 4$ with $a=\left(1-3 \phi_{\mu}\right) K_{\mu} D_{\mu}-\left(2-3 \phi_{\mu}\right) K_{m} D_{m}$ and $b=-K_{\mu} D_{\mu} K_{m} D_{m}$ where the subscripts $\mu$ and $m$ refer to the microporous and mesoporous phases, respectively (note that there are two typos in the analytical solution provided in Ref. ${ }^{18}$ ). In the above solution, only the + sign gives a physically reasonable solution.

For low density transport, $f_{k}$ is taken as the slope of the adsorption isotherm when plotted as the fluid concentration $c_{\mathrm{k}}$ in the porous material versus the fluid bulk concentration $c_{\mathrm{k}}{ }^{\mathrm{b}}$ (Henry's constant) or assumed to be 1 (no adsorption condition). Unfortunately, for other conditions, one has to use some approximations as the adsorption isotherm is not linear in such materials (Langmuir adsorption isotherm, capillary condensation, etc.). In order to be consistent with the fact that PFG NMR experiments correspond to tracer exchange, $f_{k}$ was estimated from the adsorption isotherms for the microporous and mesoporous domains as $f_{k}=$ c/c ${ }_{\mathrm{k}}^{\mathrm{b}}$. As shown in Figure 4, we obtained using Eq. (9) effective self-diffusivities $D_{\text {eff }}^{\text {EMT }}$ $8.2 \times 10^{-9} \mathrm{~m}^{2} / \mathrm{s}$. This value shows that EMT provides the right order of magnitude but overestimates the self-diffusivity of hexane in mFAU observed using PFG NMR. Using the value predicted by EMT, $D_{\text {eff }}^{\mathrm{EMT}}=8.2 \times 10^{-9} \mathrm{~m}^{2} / \mathrm{s}$, one needs to consider a network connectivity $\mathrm{z} \sim 3$ to obtain an effective self-diffusivity in agreement with the experimental value, $D_{\text {eff }}=1.4 \times 10^{-9} \mathrm{~m}^{2} / \mathrm{s}$. While this value allows reconciling the experimental and theoretical diffusivities through a mathematical adjustment of EMT, there is no physical argument to explain such a very low connectivity for a 3D system. The results above show that, despite some interesting success in modeling low density transport (gas phase) in micro/mesoporous media, the application of EMT to dense transport in hierarchical media is not straightforward. In particular, the definition of the equilibrium constant is non-trivial (i.e. except in Henry's regime where $f_{k}$ is readily obtained from the adsorption isotherms). 


\section{Conclusion}

This paper aimed at investigating adsorption and transport in hierarchical porous materials which combine different porosity scales. Using adsorption and nuclear magnetic resonance experiments, we determined the role of hierarchical interconnectivity by considering hexane adsorption and diffusion at room temperature in mesoporous zeolites with well-defined micro and mesoporosities. As an important advantage of using such model samples, we show that adsorption in such heterogeneous complex systems can be accurately described using a linear combination of the adsorbed amounts taken at the same pressure for the parent solids (FAU zeolite and Al-MCM-41 mesoporous silica). This result shows that gas adsorption, without being combined to other characterization tools, does not allow distinguishing a true hierarchical material from a material made of independent micro and mesoporosities.

In a second step, using the quantitative knowledge of the adsorbed amounts in each porosity scale, we were able to assess the ability of PFG NMR to probe interconnectivity in hierarchical porous materials. By comparing the data for mesoporous zeolites with those for pure zeolite, pure mesoporous silica, and a mechanical mixture of both, we show that this technique provides an unambiguous tool to assess interconnectivity in micro/mesoporous zeolites. The self-diffusivity for hierarchical zeolites is found to be intermediate between those for the pure zeolite and pure mesoporous silica. The additional mesoporosity in the zeolite particles increases the diffusivity by a factor of about 6 compared to that for the zeolite (in contrast, the diffusivity in the mesoporous zeolite is about 9 times slower than that for the pure mesoporous silica). These results, which are consistent with the known beneficial effect of such hierarchical connectivity on adsorption and separation processes in hierarchical 
porous media, contrasts with the picture emerging from transport in mixtures of independent zeolite and mesoporous silica (for these samples, the micro- and mesoporosities are unconnected so that molecule exchange on the timescale probed is negligible and PFG NMR probes two independent diffusion contributions corresponding to each porosity scale). Our analysis therefore shows that PFG NMR allows distinguishing true hierarchical materials from a mechanical mixture made of independent micro and mesoporous materials.

We also discuss the ability of phenomenological models available in the literature to capture the inherent complexity of fluid transport in hierarchical media such as mesoporous zeolites. Despite some interesting successes (such as in the case of gas transport), these models relying on analogies with transport in electrical circuits do not provide a rational and unifying description of the effective fluid diffusivity in hierarchical materials (although they capture the right order of magnitude). The diffusivity in hierarchical materials is lower than the average diffusivity of independent domains, which clearly evidences the presence of connectivities. This type of hierarchical model materials with well-defined micro and mesoporosity combined to adsorption and PFG NMR measurements can be the first brick to help find and probe diffusivity theories in hierarchical media.

Acknowledgments. The authors acknowledge the French National Research Agency (ANR) for supporting this study through the convention ANR ECOTECH 2010 project PETZECO (1081C0230/ANR-10-ECOT-011-03). The authors thank Francesco Di Renzo and Suresh Bhatia for helpful discussions. 
Supporting Information. Structural data including nitrogen adsorption isotherm, scanning electron microscopy, and X-Ray diffraction and nuclear magnetic resonance sequence (PDF). This information is found free of charge on the internet at http://pubs.acs.org.

\section{References.}

(1) Corma, A. From Microporous to Mesoporous Molecular Sieve Materials and Their Use in Catalysis. Chem. Rev. 1997, 97, 2373-2419.

(2) Ivanova, I. I.; Knyazeva, E. E. Micro-Mesoporous Materials Obtained by Zeolite Recrystallization: Synthesis, Characterization and Catalytic Applications. Chem. Soc. Rev. 2013, 42, 3671-3688.

(3) Soler-Illia, G. J. A. A.; Sanchez, C.; Lebeau, B.; Patarin, J. Chemical Strategies to Design Textured Materials: From Microporous and Mesoporous Oxides to Nanonetworks and Hierarchical Structures. Chem. Rev. 2002, 102, 4093-4138.

(4) Van Donk, S.; Janssen, A. H.; Bitter, J. H.; de Jong, K. P. Generation, Characterization, and Impact of Mesopores in Zeolite Catalysts. Catal. Rev. 2003, 45, 297-319.

(5) Li, K.; Valla, J.; Garcia-Martinez, J. Realizing the Commercial Potential of Hierarchical Zeolites: New Opportunities in Catalytic Cracking. ChemCatChem 2014, $6,46-66$.

(6) Prasomsri, T.; Jiao, W.; Weng, S. Z.; Garcia Martinez, J. Mesostructured Zeolites: Bridging the Gap between Zeolites and MCM-41. Chem. Commun. 2015, 51, 89008911.

(7) Wang, G.; Coppens, M. O. Rational Design of Hierarchically Structured Porous Catalysts for Autothermal Reforming of Methane. Chem. Eng. Sci. 2010, 65, 23442351.

(8) Coppens, M. Scaling-up and -down in a Nature-Inspired Way. Ind. Eng. Chem. Res. 2005, 44, 5011-5019.

(9) Garcia-Martinez, J.; Xiao, C.; Cychosz, K. A.; Li, K.; Wan, W.; Zou, X.; Thommes, M. Evidence of Intracrystalline Mesostructured Porosity in Zeolites by Advanced Gas Sorption, Electron Tomography and Rotation Electron Diffraction. ChemCatChem 2014, 6, 3110-3115.

(10) Kärger, J.; Valiullin, R. Mass Transfer in Mesoporous Materials: The Benefit of Microscopic Diffusion Measurement. Chem. Soc. Rev. 2013, 42, 4172-4197.

(11) Chmelik, C.; Enke, D.; Galvosas, P.; Gobin, O.; Jentys, A.; Jobic, H.; Kärger, J.; Krause, C. B.; Kullmann, J.; Lercher, J.; et al. Nanoporous Glass as a Model System 
for a Consistency Check of the Different Techniques of Diffusion Measurement. ChemPhysChem 2011, 12, 1130-1134.

(12) Valiullin, R.; Naumov, S.; Galvosas, P.; Kärger, J.; Woo, H.-J.; Porcheron, F.; Monson, P. a. Exploration of Molecular Dynamics during Transient Sorption of Fluids in Mesoporous Materials. Nature 2006, 443, 965-968.

(13) Adem, Z.; Guenneau, F.; Springuel-Huet, M. A.; Gédéon, A.; Iapichella, J.; Cacciaguerra, T.; Galarneau, A. Diffusion Properties of Hexane in Pseudomorphic MCM-41 Mesoporous Silicas Explored by Pulsed Field Gradient NMR. J. Phys. Chem. C 2012, 116, 13749-13759.

(14) Mehlhorn, D.; Valiullin, R.; Kärger, J.; Cho, K.; Ryoo, R. Exploring the Hierarchy of Transport Phenomena in Hierarchical Pore Systems by NMR Diffusion Measurement. Microporous Mesoporous Mater. 2012, 164, 273-279.

(15) Mehlhorn, D.; Inayat, A.; Schwieger, W.; Valiullin, R.; Kärger, J. Probing Mass Transfer in Mesoporous Faujasite-Type Zeolite Nanosheet Assemblies. ChemPhysChem 2014, 15, 1681-1686.

(16) Kärger, J. Transport Phenomena in Nanoporous Materials. Chemphyschem 2014, 1-29.

(17) Zeigermann, P.; Naumov, S.; Mascotto, S.; Kärger, J.; Smarsly, B. M.; Valiullin, R. Diffusion in Hierarchical Mesoporous Materials: Applicability and Generalization of the Fast-Exchange Diffusion Model. Langmuir 2012, 28, 3621-3632.

(18) Bonilla, M. R.; Valiullin, R.; Kärger, J.; Bhatia, S. K. Understanding Adsorption and Transport of Light Gases in Hierarchical Materials Using Molecular Simulation and Effective Medium Theory. J. Phys. Chem. C 2014, 118, 14355-14370.

(19) Kirkpatrick, S. Percolation Ancl Conduction. Rev. Mod. Phys. 1973, 45, 475-588.

(20) Bruggeman, D. A. G. Berechnung Verschiedener Physikalischer Konstanten von Heterogenen Substanzen. Ann. Phys. 1935, 24, 636.

(21) Hibbe, F.; Chmelik, C.; Heinke, L.; Pramanik, S.; Li, J.; Ruthven, D. M.; Tzoulaki, D.; Kärger, J. The Nature of Surface Barriers on Nanoporous Solids Explored by Microimaging of Transient Guest Distributions. J. Am. Chem. Soc. 2011, 133, 28042807.

(22) Heinke, L.; Kärger, J. Correlating Surface Permeability with Intracrystalline Diffusivity in Nanoporous Solids. Phys. Rev. Lett. 2011, 106, 074501.

(23) Galarneau, A.; Iapichella, J.; Bonhomme, K.; De Renzo, F.; Kooyman, P.; Terasaki, O.; Fajula, F. Controlling the Morphology of Mesostructured Silicas by Pseudomorphic Transformation: A Route towards Applications. Adv. Funct. Mater. 2006, 16, 16571667. 
(24) Galarneau, A.; Desplantier, D.; Dutartre, R.; Di Renzo, F. Micelle-Templated Silicates as a Test Bed for Methods of Mesopore Size Evaluation. Microporous Mesoporous Mater. 1999, 27, 297-308.

(25) Galarneau, A.; Villemot, F.; Rodriguez, J.; Fajula, F.; Coasne, B. Validity of the $\mathrm{T}$ - Plot Method to Assess Microporosity in Hierarchical Micro / Mesoporous Materials. Langmuir 2014, 30, 13266-13274.

(26) Adem, Z.; Guenneau, F.; Springuel-Huet, M. A.; Gédéon, A. PFG NMR Investigation of Hydrocarbon Diffusion in Large NaX Zeolite Crystals: Effect of Internal Field Gradients on Diffusion Data. Microporous Mesoporous Mater. 2008, 114, 337-342.

(27) Cotts, R. .; Hoch, M. J. .; Sun, T.; Markert, J. . Pulsed Field Gradient Stimulated Echo Methods for Improved NMR Diffusion Measurements in Heterogeneous Systems. $J$. Magn. Reson. 1989, 83 (2), 252-266.

(28) Weber, G.; Simonot-Grange, M. H. Characterization of the Dealumination Effect into H-Faujasites by Adsorption .2. The Hexane Molecule as a Pore Volume Probe. Zeolites 1994, 14, 433-438.

(29) Coasne, B.; Galarneau, A.; Gerardin, C.; Fajula, F.; Villemot, F. Molecular Simulation of Adsorption and Transport in Hierarchical Porous Materials. Langmuir 2013, 29, 7864-7875.

(30) Villemot, F.; Galarneau, A.; Coasne, B. Adsorption and Dynamics in Hierarchical Metal Organic Frameworks. J. Phys. Chem. C 2014.

(31) Beckert, S.; Stallmach, F.; Toufar, H.; Freude, D. Tracing Water and Cation Diffusion in Hydrated Zeolites of Type Li-. J. Phys. Chem. C 2013, 117, 24866-24872.

(32) Mehlhorn, D.; Valiullin, R.; Kärger, J.; Schumann, K.; Brandt, A.; Unger, B. Transport Enhancement in Binderless Zeolite X- and A-Type Molecular Sieves Revealed by PFG NMR Diffusometry. Microporous Mesoporous Mater. 2014, 188, 126-132.

(33) Smit, B.; Maesen, T. L. M. Molecular Simulations of Zeolites: Adsorption, Diffusion, and Shape Selectivity. Chem. Rev. 2008, 108, 4125-4184.

(34) Gheorghiu, S.; Coppens, M. O. Optimal Bimodal Pore Networks for Heterogeneous Catalysis. AIChE J. 2004, 50, 812-820.

(35) Bocquet, L.; Charlaix, E. Nanofluidics, from Bulk to Interfaces. Chem. Soc. Rev. 2010, 39, 1073-1095.

(36) Jobic, H.; Theodorou, D. N. Quasi-Elastic Neutron Scattering and Molecular Dynamics Simulation as Complementary Techniques for Studying Diffusion in Zeolites. Microporous Mesoporous Mater. 2007, 102, 21-50.

(37) Zeigermann, P.; Kärger, J.; Valiullin, R. Diffusion in Microporous Materials with Embedded Mesoporosities. Microporous Mesoporous Mater. 2013, 178, 84-89. 


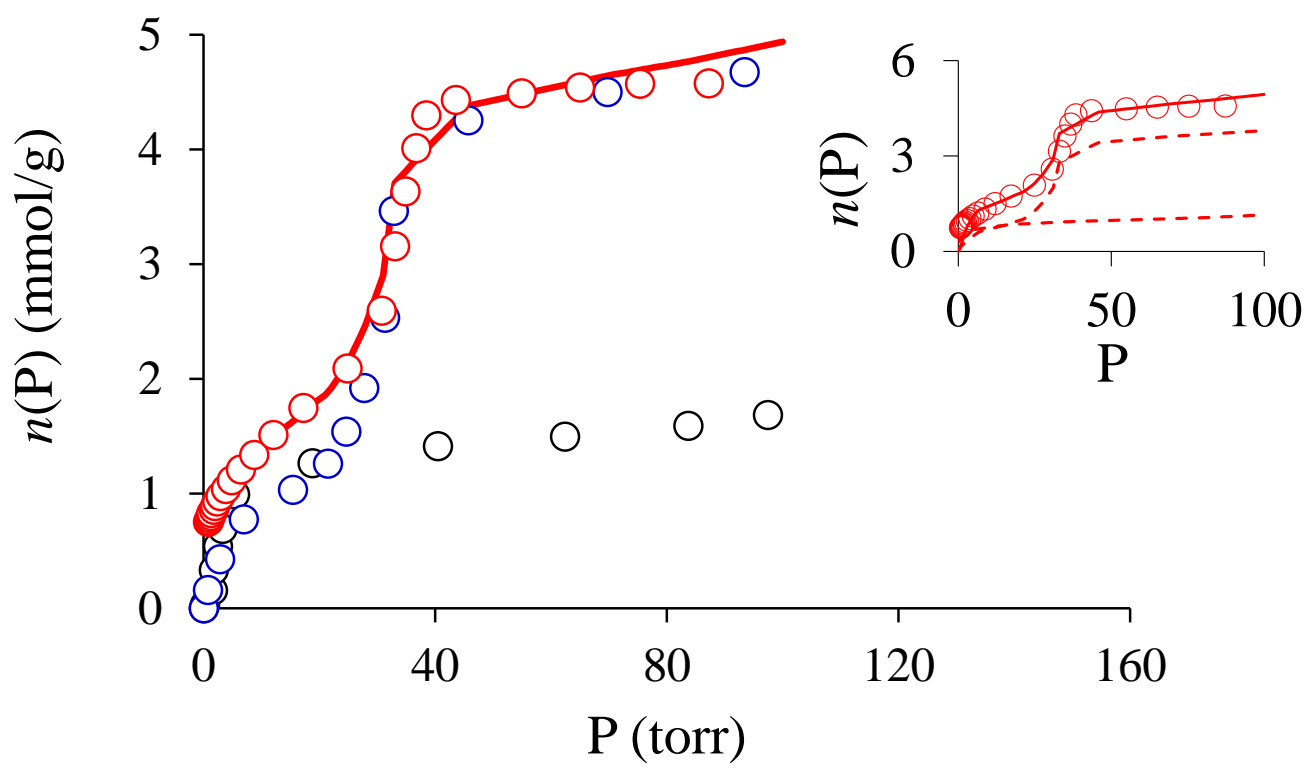

Figure 1. (color online) Hexane adsorption isotherm at $298 \mathrm{~K}$ in Faujasite zeolite (black o), Al-MCM-41 (blue ○), and mesoporous FAU (red $\circ$ ). The solid line is a fit of the adsorption isotherm for mesoporous FAU using the superimposition principle (see text). The inset shows the adsorption isotherm for mFAU (open circles) together with the linear combination (solid line). The dashed lines show the microporous and mesoporous contributions of the adsorption isotherm for mFAU. 

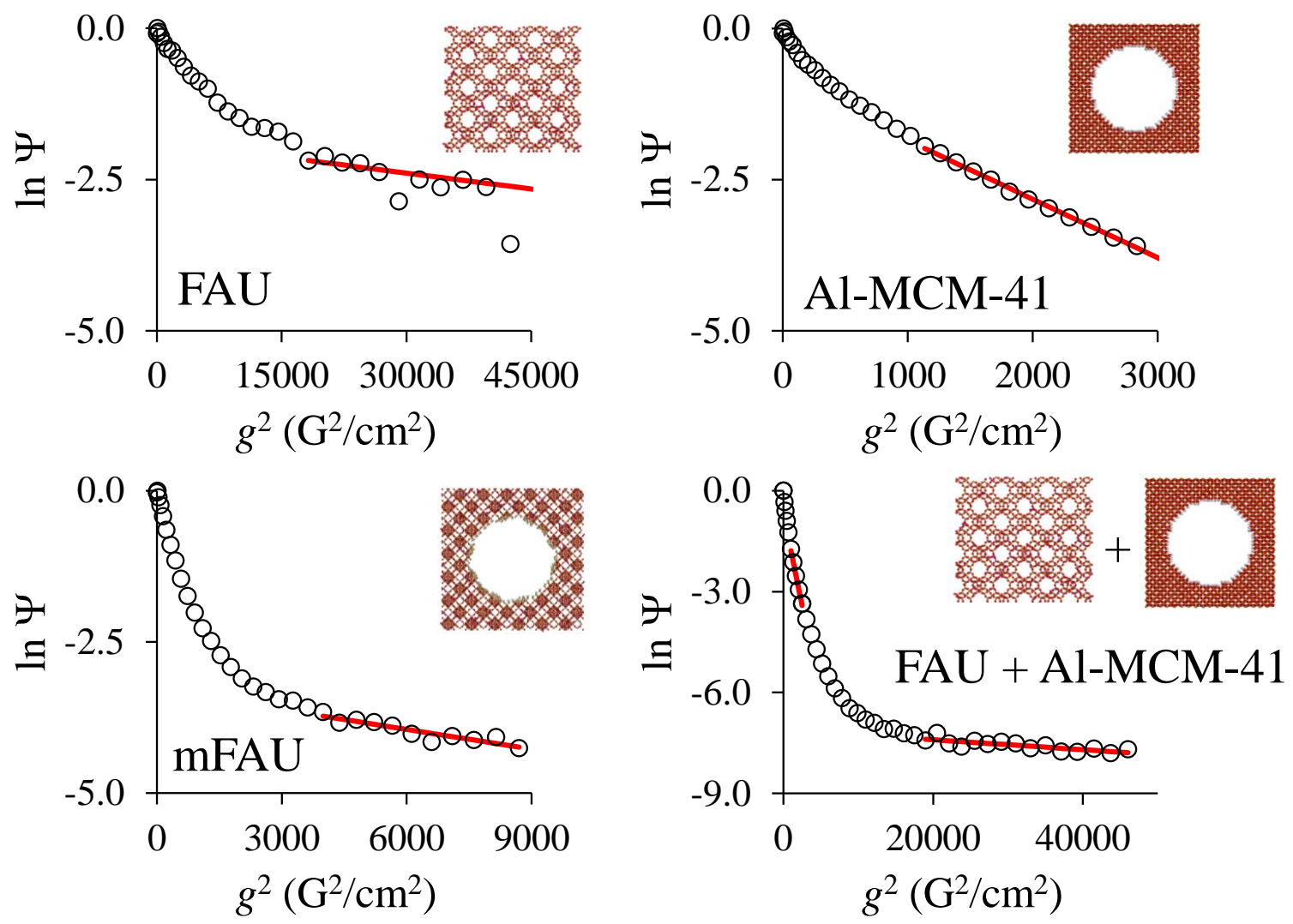

Figure 2. Attenuation $\Psi$ of the spin echo signal as a function of the gradient strength $g$ measured from PFG NMR for hexane adsorbed in FAU (top left), Al-MCM-41 (top right), mFAU (bottom left) and a mechanical mixture of FAU+Al-MCM-41 (bottom right). For each material, the hexane adsorbed amount was imposed to correspond to a loading of $60 \%$. The red lines are linear fits of the data in the high field gradient $\mathrm{g}$ range $\left(\mathrm{g}^{2}>10^{3} \mathrm{G}^{2} \cdot \mathrm{cm}^{-2}\right)$. For the mechanical mixture, two fits were performed to determine the diffusion coefficient in the microporosity (high field gradient) and in the mesoporosity (low field gradient). For the mesoporosity part, the diffusivity has been calculated from the slope of the decay after substracting from the decay the contribution stemming from FAU (i.e. the extrapolation of the slowly decaying part back to the origin). 


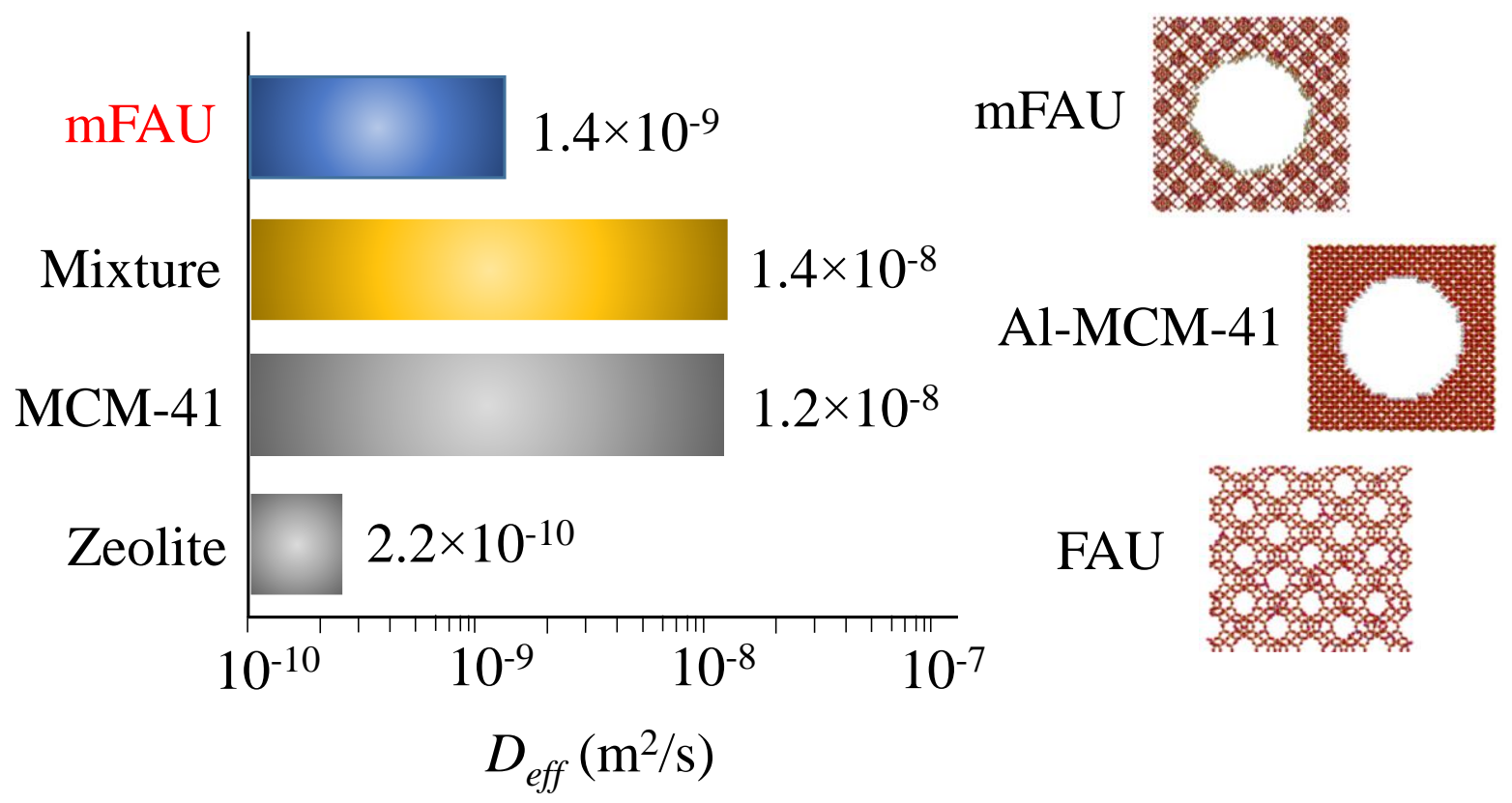

Figure 3. Effective diffusivity $D_{\text {eff }}$ as determined from PFG NMR for hexane adsorbed at 298 $\mathrm{K}$ in FAU, Al-MCM-41, mFAU and a mechanical mixture of FAU+Al-MCM-41. For the mixture, the self-diffusivity corresponds to the fast contribution (Al-MCM-41). Note that the diffusivities $D_{\text {eff }}$ are in a $\log$ scale for the sake of clarity. 


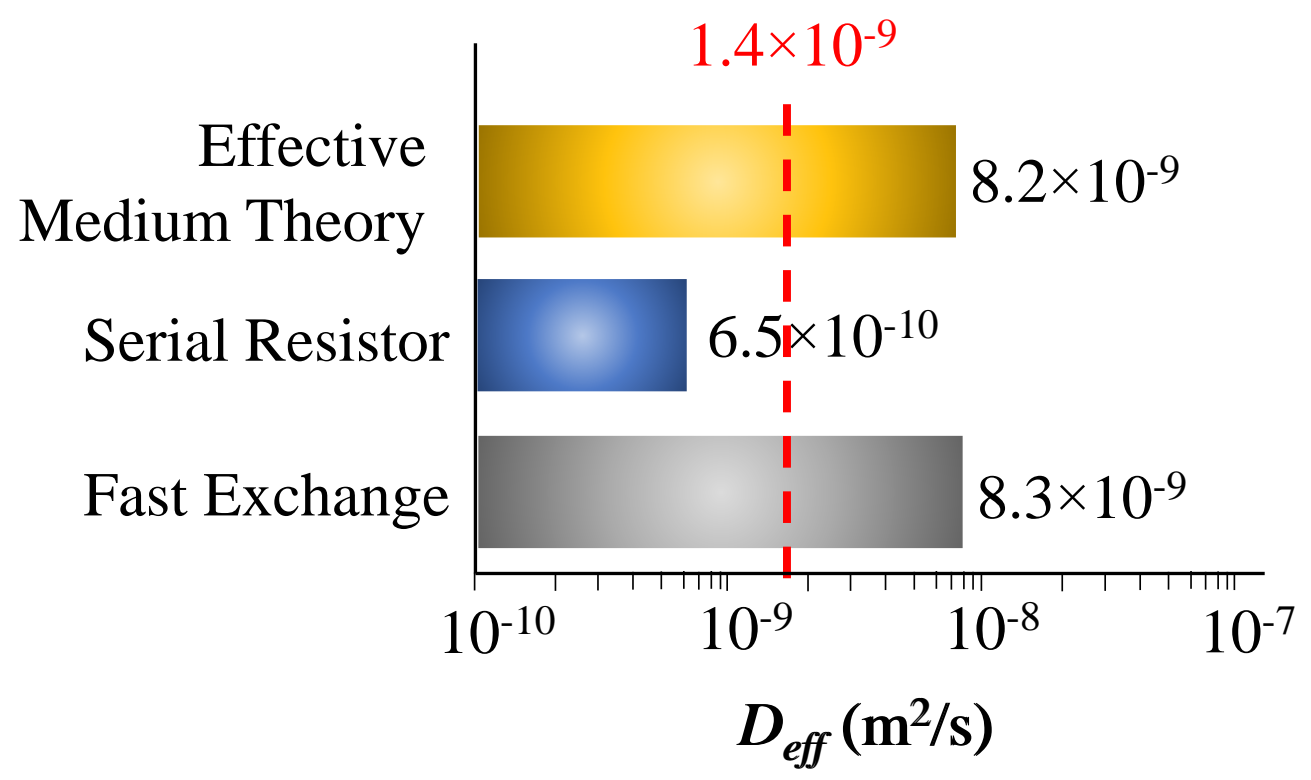

Figure 4. Predicted effective diffusivities $D_{\text {eff }}$ for the different models considered in this work: fast exchange, serial resistor network, and effective medium theory. The dashed line indicates the experimental self-diffusivity of hexane in mFAU $D_{\text {eff }}$ as obtained from PFG NMR. Note that the diffusivities $D_{\text {eff }}$ are in a log scale for the sake of clarity. 


\section{TOC Figure}

\section{Probing Interconnectivity in Hierarchical Microporous/Mesoporous}

Materials using Adsorption and Nuclear Magnetic Resonance Diffusion

Anne Galarneau, ${ }^{\dagger} * *$ Flavien Guenneau, ${ }^{\ddagger}$ Antoine Gedeon, ${ }^{\ddagger}$ Diaa Mereib,${ }^{\ddagger}$ Jeremy Rodriguez, ${ }^{\dagger}$ François Fajula, ${ }^{\dagger}$ Benoit Coasne ${ }^{\dagger, \S, *}$

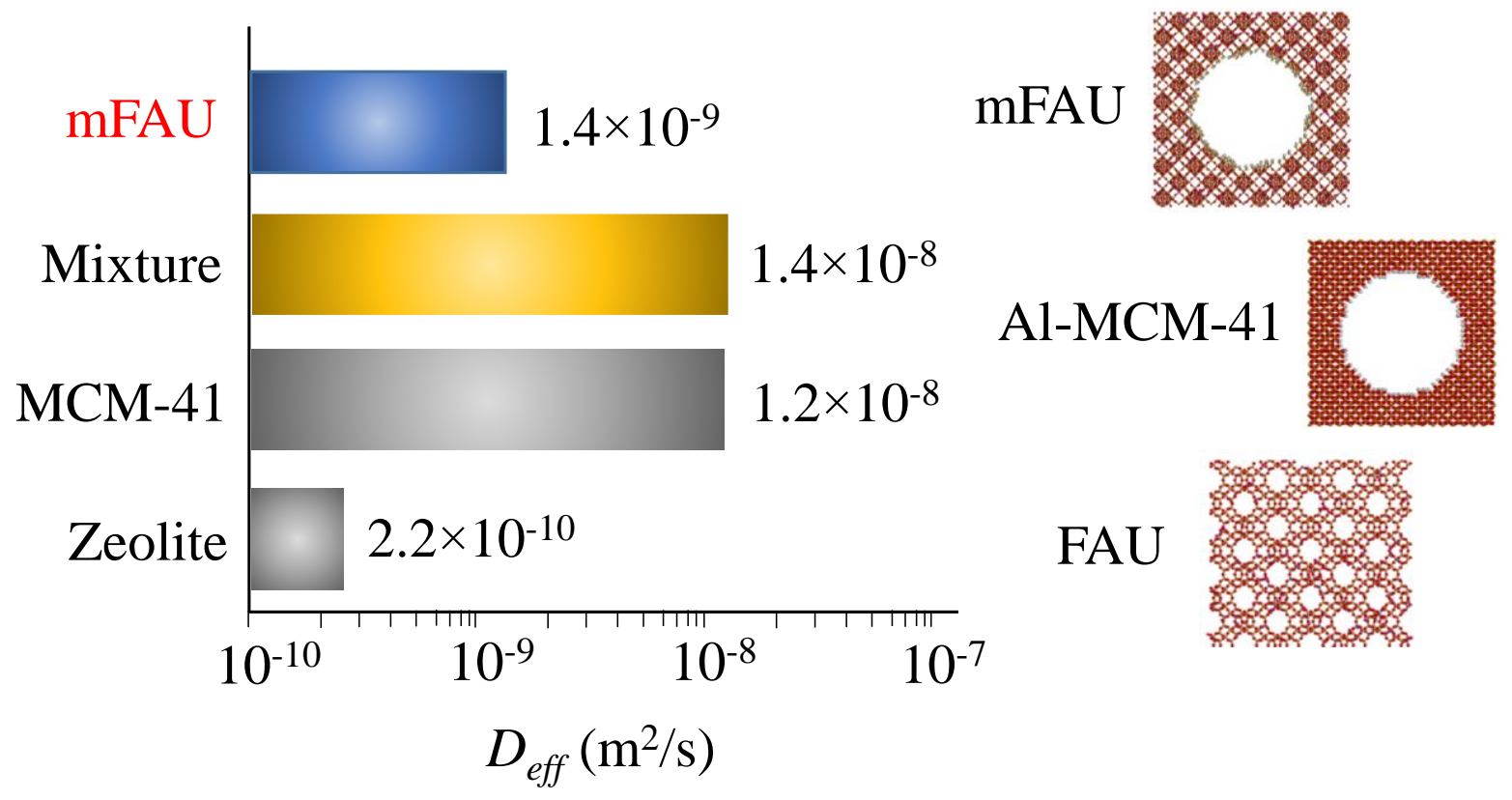

